\title{
Myocardial blood flow quantitation with the SPECT technique: Where do we stand?
}

\author{
Wei Fang, MD, PhD, a and Bailing Hsu, $\mathrm{PhD}^{\mathrm{b}}$ \\ a Department of Nuclear Medicine, Fuwai Hospital, National Center for Cardiovascular Diseases, \\ Chinese Academy of Medical Sciences and Peking Union Medical College, Beijing, China \\ b Nuclear Science and Engineering Institute, University of Missouri-Columbia, Columbia, MO
}

Received Aug 29, 2020; accepted Aug 31, 2020

doi: $10.1007 / \mathrm{s} 12350-020-02373-0$

\section{See related article, pp. 622-629}

Since 2013, there are numerous research studies (more than 30) reporting myocardial blood flow (MBF) quantitation with the SPECT technique. Some of them highlighted the capability of SPECT technique for detection of coronary artery disease (CAD) using rapid rotating gantry (RRG) cameras integrated with full physical corrections (e.g. corrections for attenuation, scatter, collimator blur and statistical noise). ${ }^{1,2}$ The rest majority reported the ability of CZT SPECT cameras with collimators of stationary multiple pinholes or sweeping parallel holes, and with or without attenuation correction (AC). ${ }^{3-8}$ Technical validations were properly achieved by head-to-head comparison with PET MBF quantitation on the same cohort or the diagnostic assessment for CAD utilizing coronary angiography (CAG) or factional flow reserve (FFR) as the reference standard. ${ }^{3-11}$ When the relevant physical interference in dynamic SPECT images was properly corrected, RRG and CZT cameras likely performed to the same level regardless of the CZT cameras possessing additional merits from higher count rate and less scatter by higher energy resolution. ${ }^{12}$ It has been reported that resting MBF and stress MBF can be highly overestimated (20\% to $40 \%$ ) for CZT cameras without AC applied as compared to PET flow quantitation. ${ }^{11,13,14}$ For RRG cameras, the impact from no AC appended to other physical factors can be even more brutal (100\% to

Reprint requests: Bailing $\mathrm{Hsu}, \mathrm{PhD}$, Nuclear Science and Engineering Institute, University of Missouri-Columbia, Columbia, $\mathrm{MO}$; bailinghsu@gmail.com

J Nucl Cardiol 2022;29:630-2.

$1071-3581 / \$ 34.00$

Copyright (C) 2020 American Society of Nuclear Cardiology.
145\%). ${ }^{15}$ Because myocardial flow reserve (MFR) or coronary flow reserve (CFR) can be relatively preserved within an acceptable range even missing AC, validation for SPECT MBF quantitation with invasive parameters (percentage of narrowed diameter in coronary artery and FFR) has been mainly focused on the single parameter, CFR (or MFR) ${ }^{3-8}$ The importance of stress MBF is still not yet explored for SPECT MBF quantitation due to the described limitation of SPECT systems and dedicated processing software.

In the study by $\mathrm{Li}$ et al., ${ }^{16} \mathrm{CFR}$ exhibited much better diagnostic value than stress MBF in defining significant hemodynamic coronary stenosis suggested by the area under the ROC curve (AUC) for the diagnosis of abnormal FFR (CFR: 0.864 vs stress MBF: 0.673). Although authors did not further investigate this particular finding in their study, comparing the difference in diagnostic performance of these two quantitative values, stress MBF and CFR, is of physiological importance. So far, there still exists debate as to the integration of best index from quantitative flow analysis. CFR has been shown to add incremental value for prognosis ${ }^{17}$; however, it seems that stress MBF can be superior to CFR for diagnosing hemodynamically significant $\mathrm{CAD}$ in previous quantitative cardiac PET studies. ${ }^{18-20}$ If stress MBF is sufficient for the diagnostic purpose, stress-only protocol with less radiation dose and shorter acquisition time should be more welcome. Because the calculation of CFR depends on both resting and stress MBF, diminished stress MBF does not necessarily cause a reduction in MFR if the resting MBF is in a very low level. While patients with a history of myocardial infarction having low resting MBF may still exhibit normal CFR (or MFR) values (Figure 1). ${ }^{21}$ As reported by the study, there was an inverse trend towards higher stress MBF in coronary arteries with stenoses than in vessels without stenoses while there was no significant difference in CFR between stenotic and non-stenotic 


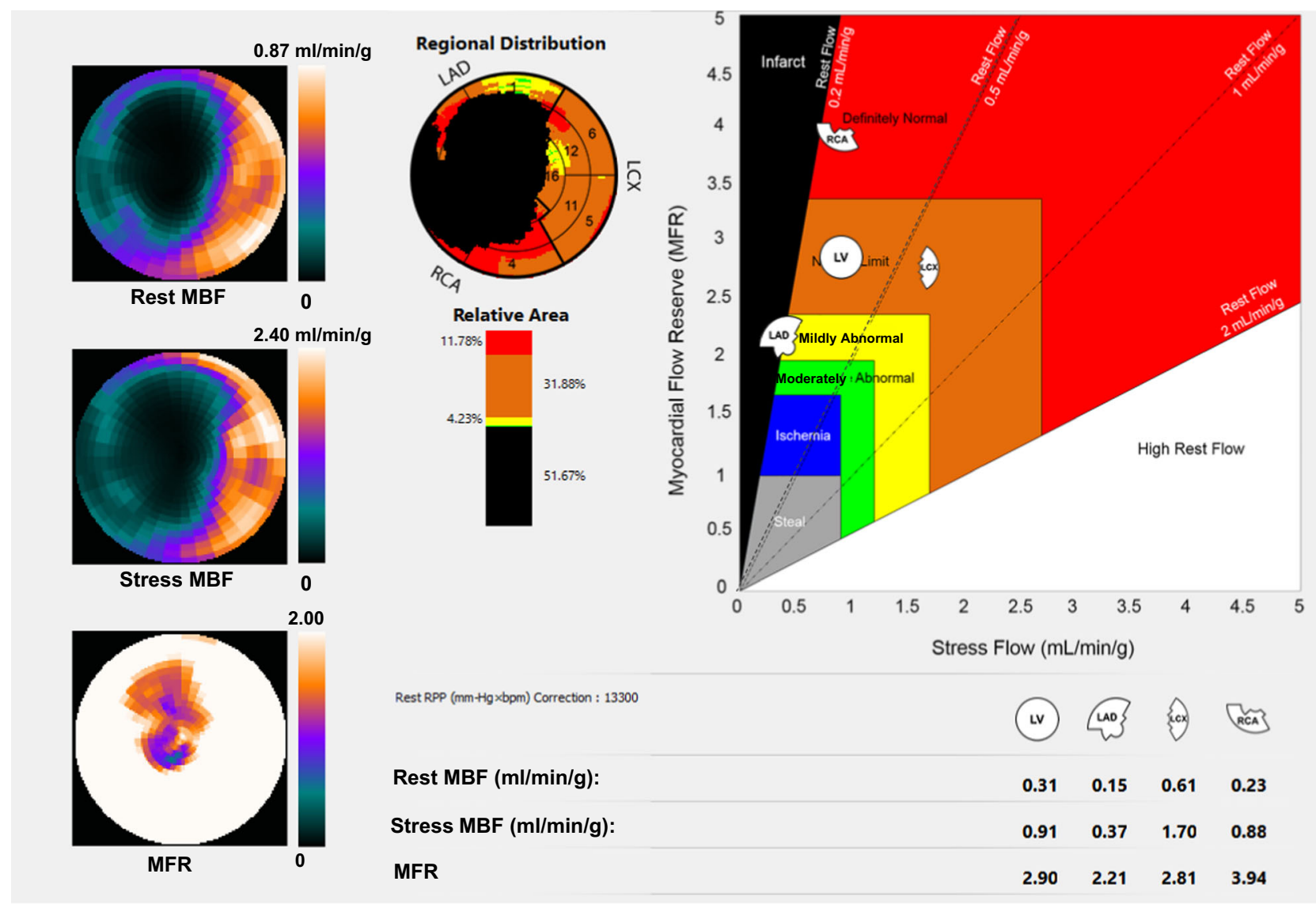

Figure 1. A 70-year male patient with a history of old myocardial infarction. Both his rest MBF and stress MBF were relatively low $(0.31,0.91 \mathrm{~mL} / \mathrm{min} / \mathrm{g})$ to yield a high MFR (2.90). Extent of the infarct was $51.67 \% .^{21}$.

arteries. Lacking of AC should be recognized as the critical issue to affect the calculation of absolute MBF in their study. It is indeed a downside for the model of CZT SPECT camera utilized by Li et al. without the option to perform AC. Furthermore, only small sample size to evaluate the diagnostic accuracy of quantitative SPECT flow measurement using invasive FFR as the reference standard may exist statistical bias in their study. The work of $\mathrm{Li}$ et al. only included 33 intermediate lesions with FFR assessment. Nonetheless, good diagnostic performance in terms of sensitivity, specificity, and accuracy as $88.9 \%, 83.3 \%$, and $87.9 \%$ for SPECTderived CFR was still observed. From the Li's study, it is markable that SPECT-derived CFR may be useful to determine hemodynamical significance for intermediate lesions as the current practical utilization of invasive FFR still focuses to resolve this unanswered zone by CAG. However, the study was ineligible for further subgroup analysis on the discordance between CFR and FFR as authors commented. It is well known that there exists concordance and discordance between CFR and
FFR under various pathophysiological conditions, and discordance between CFR and FFR does not necessarily represent the mistake of CFR. ${ }^{22}$ For example, diffuse atherosclerosis or small vessel disease may not cause localized pressure gradient in FFR but present a reduction in CFR. FFR is now recommended as the golden standard for revascularization decision-making for $\mathrm{CAD}$; nevertheless, combined pressure and flow measurement to guide treatment of coronary stenoses is worthwhile investigating. Since SPECT-derived CFR has showed good correlation and accuracy with invasive FFR by Li's work and other studies, large prospective studies in collaborative centers are still needed to warrant the potential role of quantitative SPECT flow measurement for both stress MBF and CFR as the gatekeeper for invasive FFR or even guiding clinical decision for revascularization for patients with CAD.

\section{Disclosures}

Drs. Fang and Hsu have nothing to disclose. 


\section{References}

1. Klein R, Hung GU, Wu TC, Huang WS, Li D, deKemp R, et al. Feasibility and operator variability of myocardial blood flow and reserve measurements with ${ }^{99 \mathrm{~m}} \mathrm{Tc}$-sestamibi quantitative dynamic SPECT/CT imaging. J Nucl Cardiol 2014;21:1075-88.

2. Hsu B, Chen FC, Wu TC, Huang WS, Hou PN, Chen CC, et al. Quantitation of myocardial blood flow and myocardial flow reserve with $99 \mathrm{mTc}$-sestamibi dynamic SPECT/CT to enhance detection of coronary artery disease. Eur J Nucl Med Mol Imaging 2014;41:2294-306.

3. Iguchi N, Utanohara Y, Suzuki Y, et al. Myocardial flow reserve derived by dynamic perfusion single-photon emission computed tomography reflects the severity of coronary atherosclerosis. Int J Cardiovasc Imaging 2018;34:1493-501.

4. de Souza ACDAH, Gonçalves BKD, Tedeschi AL, Lima RSL. Quantification of myocardial flow reserve using a gamma camera with solid-state cadmium-zinc-telluride detectors: Relation to angiographic coronary artery disease [published online ahead of print, 2019 Jun 20]. J Nucl Cardiol. 2019;10.

5. Shiraishi S, Tsuda N, Sakamoto F, et al. Clinical usefulness of quantification of myocardial blood flow and flow reserve using CZT-SPECT for detecting coronary artery disease in patients with normal stress perfusion imaging. J Cardiol 2020;75:400-9.

6. Ben Bouallègue F, Roubille F, Lattuca B, et al. SPECT myocardial perfusion reserve in patients with multivessel coronary disease: Correlation with angiographic findings and invasive fractional flow reserve measurements. J Nucl Med 2015;56:1712-7.

7. Zavadovsky KV, Mochula AV, Boshchenko AA, et al. Absolute myocardial blood flows derived by dynamic CZT scan vs invasive fractional flow reserve: Correlation and accuracy [published online ahead of print, 2019 Mar 7]. J Nucl Cardiol 2019

8. Hyafil F, Chequer R, Sorbets E, et al. Head-to-head comparison of the diagnostic performances of Rubidium-PET and SPECT with CZT camera for the detection of myocardial ischemia in a population of women and overweight individuals. J Nucl Cardiol 2020;27:755-68

9. Hsu B, Hu LH, Yang BH, et al. SPECT myocardial blood flow quantitation toward clinical use: A comparative study with $13 \mathrm{~N}$ Ammonia PET myocardial blood flow quantitation. Eur J Nucl Med Mol Imaging 2017;44:117-28.

10. Wells RG, Marvin B, Poirier M, Renaud J, deKemp RA, Ruddy TD. Optimization of SPECT measurement of myocardial blood flow with corrections for attenuation, motion, and blood binding compared with PET. J Nucl Med 2017;58:2013-9.

11. Comparison between N13NH3-PET and 99mTc-TetrofosminCZT SPECT in the evaluation of absolute myocardial blood flow and flow reserve [published online ahead of print, 2019 Nov 14]. J Nucl Cardiol 2019;10.
12. Ma R, Wang L, Wu D, et al. Myocardial blood flow quantitation in patients with congestive heart failure: Head-to-head comparison between rapid-rotating gantry SPECT and CZT SPECT [published online ahead of print, 2019 Feb 19]. J Nucl Cardiol 2019.

13. Agostini D, Roule V, Nganoa C, et al. First validation of myocardial flow reserve assessed by dynamic $99 \mathrm{mTc}$-sestamibi CZT-SPECT camera: Head to head comparison with 15O-water PET and fractional flow reserve in patients with suspected coronary artery disease. The WATERDAY study. Eur J Nucl Med Mol Imaging 2018;45:1079-90.

14. Bailly M, Thibault F, Courtehoux M, Metrard G, Ribeiro MJ. Impact of attenuation correction for CZT-SPECT measurement of myocardial blood flow [published online ahead of print, $2020 \mathrm{Feb}$ 20]. J Nucl Cardiol.

15. Wang L, Wu D, Yang Y, et al. Avoiding full corrections in dynamic SPECT images impacts the performance of SPECT myocardial blood flow quantitation. J Nucl Cardiol 2017;24:133246.

16. Li C, Xu R, Yao K, Zhang J, Chen S, Pang L, et al. Functional significance of intermediate coronary stenosis in patients with single-vessel coronary artery disease: A comparison of dynamic SPECT coronary flow reserve with intracoronary pressure-derived Fractional Flow Reserve (FFR) J Nucl Cardiol 2020.

17. Herzog BA, Husmann L, Valenta I, et al. Long-term prognostic value of $13 \mathrm{~N}$-ammonia myocardial perfusion positron emission tomography added value of coronary flow reserve. J Am Coll Cardiol 2009;54:150-6.

18. Danad I, Uusitalo V, Kero T, et al. Quantitative assessment of myocardial perfusion in the detection of significant coronary artery disease: Cutoff values and diagnostic accuracy of quantitative [(15)O $\mathrm{H}_{2} \mathrm{O}$ PET imaging. J Am Coll Cardiol 2014;64:1464-75.

19. Joutsiniemi E, Saraste A, Pietilä M, et al. Absolute flow or myocardial flow reserve for the detection of significant coronary artery disease? Eur Heart J Cardiovasc Imaging 2014;15:659-65.

20. Moody JB, Poitrasson-Rivière A, Hagio T, et al. Added value of myocardial blood flow using ${ }^{18} \mathrm{~F}$-flurpiridaz PET to diagnose coronary artery disease: The flurpiridaz 301 trial. J Nucl Cardiol 2020. https://doi.org/10.1007/s12350-020-02034-2.

21. Chen LC, Hung HF, Jong BH, et al. A method to measure the extent of myocardial ischemia and steal with SPECT myocardial blood flow quantitation. Ann Nucl Med 2020;34:682-90.

22. Johnson NP, Kirkeeide RL, Gould KL. Is discordance of coronary flow reserve and fractional flow reserve due to methodology or clinically relevant coronary pathophysiology? JACC Cardiovasc Imaging 2012;5:193-202.

Publisher's Note Springer Nature remains neutral with regard to jurisdictional claims in published maps and institutional affiliations. 